\title{
Health Indicator Bacteria That Is Useful for Risk Assessment of Peri-Implantitis
}

\author{
Hidenori Suzuki ${ }^{1}$, Osamu Tsuzukibashi², Akira Fukatsu ${ }^{2 *}$ \\ ${ }^{1}$ Department of Laboratory Medicine for Dentistry, Nihon University Graduate School of Dentistry at Matsudo, Chiba, Japan \\ ${ }^{2}$ Department of Laboratory Medicine and Dentistry for the Compromised Patient, Nihon University School of Dentistry, \\ Matsudo, Chiba, Japan \\ Email: ${ }^{\star}$ fukatsu.akira@nihon-u.ac.jp
}

How to cite this paper: Suzuki, H., Tsuzukibashi, O. and Fukatsu, A. (2021) Health Indicator Bacteria That Is Useful for Risk Assessment of Peri-Implantitis. Open Journal of Stomatology, 11, 360-372. https://doi.org/10.4236/ojst.2021.119032

Received: August 3, 2021

Accepted: September 15, 2021

Published: September 18, 2021

Copyright (c) 2021 by author(s) and Scientific Research Publishing Inc. This work is licensed under the Creative Commons Attribution International License (CC BY 4.0). http://creativecommons.org/licenses/by/4.0/

\begin{abstract}
We established a novel identification method for oral Rothia species using one-step multiplex PCR analysis to investigate whether the monitoring of oral Rothia species levels is useful for peri-implantitis risk assessment, and to examine the oxygen concentration that these organisms need for growth in vitro. The mean number and proportion of Rothia aeria in peri-implant sulcus fluid (PISF) samples was significantly higher in the healthy implant group than in the peri-implantitis group $(\mathrm{P}<0.05)$. Moreover, $R$. aeria under aerobic conditions vigorously grew compared with those under anaerobic conditions, and this organism grew only at the upper layer where high oxygen concentrations existed in a semi-liquid nutrient medium. Therefore, the monitoring of $R$. aeria levels may be suitable as an indicator of healthy peri-implant tissue conditions for the prevention of peri-implantitis.
\end{abstract}

\section{Keywords}

Peri-Implantitis, Multiplex PCR, Rothia aeria, Oral Cavity

\section{Introduction}

The successful use of jawbone-anchored (osseointegrated) titanium dental implants for the rehabilitation of edentulous and partially dentate patients has been well documented [1]-[7]. The results of implant treatment have mostly been satisfactory with survival rates of $85 \%$ to $99 \%$. However, infections such as implant mucositis and peri-implantitis occur around dental implants [8] [9] [10] [11] [12]. The prevalence of peri-implant infections, defined as bone loss $\geq 3.1 \mathrm{~mm}$ compared with one-year radiographic data after placement of the supra-structure, and bleeding on probing (BOP) approaches $20 \%$ of cases within a 15 -year follow-up 
period [13]. Patients with a history of periodontitis also appear to be more susceptible to developing peri-implant infections [14]. Smoking is another risk factor that has been associated with peri-implant infections [15] [16] [17] [18].

Shortly after the installation of titanium implants, an implant sub-mucosal microbiota is established [19]. In fact, the initial colonization of peri-implant pockets with bacteria associated with periodontitis has been demonstrated to occur within two weeks [20]. This early colonization pattern may contribute to the development of peri-implant lesions. Leonhardt et al. [21] reported that peri-implantitis lesions contain not only periodontopathic bacteria but also staphylococci, enteric species, and yeasts, indicating that a complex microbiota is associated with the infections of tissues surrounding implants. Such observations are consistent with the hypothesis that an extensive unknown microbiota may be associated with periodontitis [22].

Currently, bacteriological examinations of implant treatments target periodontopathic bacteria such as red complex bacteria, including Porphyromonas gingivalis, and detect them qualitatively or quantitatively. However, those examinations do not reflect the peri-implant tissue conditions precisely, because periodontopathic bacteria may be detected from healthy peri-implant sites [23]. Consequently, we concluded that it is suboptimal to use periodontopathic bacteria as an unhealthy indicator, and have instead explored bacteria that indicate healthy peri-implant tissue conditions. We chose oral Rothia species, which are part of normal oral flora, to be examined as potential health indicators. These species include Rothia mucilaginosa, Rothia dentocariosa, and Rothia aeria [24].

In addition to periodontitis, peri-implantitis is primarily caused by bacterial infection and presents symptoms such as soft tissue inflammation and bone resorption, but often progresses asymptomatically. However, peri-implantitis rapidly progresses compared with periodontitis, and therapeutics for periodontitis have limited effectiveness against peri-implantitis [25] [26] [27]. The detachment of the implant body in severe peri-implantitis cases occurs by resorption of the supporting bone, thereby reducing the quality of life of patients. In order to prevent the onset of peri-implantitis, it is necessary to establish a useful bacteriological examination system.

In the present study, we established a high-precision novel identification method for oral Rothia species using one-step multiplex PCR analysis to investigate whether oral Rothia species levels are useful for peri-implantitis risk assessment. We also examined the oxygen concentrations that this organism needs for growth in vitro. Furthermore, the relationship between red complex bacteria most involved in periodontal disease and peri-implantitis was also investigated using PCR analysis.

\section{Materials and Methods}

\subsection{Bacterial Strains and Culture Conditions}

The following bacterial strains were used in the present study: $R$. mucilaginosa 
JCM 10910, $R$. dentocariosa JCM 306, $R$. aeria JCM 11412, $R$. aeria Num-Ra7006, Rothia terrae JCM 15158, Rothia amarae JCM 11375, Rothia nasimurium JCM 10909, Rothia endophytica JCM 18541, Streptococcus mitis ATCC 49456, Streptococcus oralis ATCC 10557, Streptococcus sanguinis ATCC 10556, Streptococcus salivarius JCM 5707, Streptococcus anginosus ATCC 33397, Streptococcus mutans NCTC 10449, Actinomyces naeslundii ATCC 12104, Actinomyces oris ATCC 27044, Actinomyces odontolyticus ATCC 17929, Actinomyces israelii ATCC 12102, Neisseria sicca ATCC 29256, Corynebacterium matruchotii ATCC 14266, Corynebacterium durum ATCC 33449, and P. gingivalis ATCC 33277. Bacterial strains other than $P$. gingivalis were maintained by cultivation on Bact $^{\mathrm{TM}}$ Brain Heart Infusion (BHI, Becton, Dickinson and Co., Sparks, MD, USA) and $1.5 \%$ agar (BHI agar). All bacterial strains were cultured at $37^{\circ} \mathrm{C}$ overnight in an atmosphere of $5 \% \mathrm{CO}_{2}$ in a $\mathrm{CO}_{2}$ incubator (MCO-18AIC; Sanyo Electric Co., Tokyo, Japan). P. gingivalis was cultured at $37^{\circ} \mathrm{C}$ for $48 \mathrm{~h}$ under anaerobic conditions with a gas pack system (AnaeroPack ${ }^{\circledR}$; Mitsubishi Gas Chemical Co., Inc., Tokyo, Japan).

\subsection{Design of Species-Specific Primers for Oral Rothia Species}

The design of species-specific primers for oral Rothia species was performed as described previously [24]. Briefly, the 16S rDNA sequences of $R$. dentocariosa (accession no. M59055), R. mucilaginosa (accession no. X87758), and $R$ aeria (accession no. AB071952) were obtained from the DNA Data Bank of Japan (DDBJ; https://www.ddbj.nig.ac.jp/services.html, Mishima, Japan), and a multiplex sequence alignment analysis was performed using the CLUSTALW program; i.e., the $16 \mathrm{~S}$ rDNA sequences of seven Rothia species were aligned and analyzed. Homologies among the primers selected for $R$. dentocariosa, $R$. mucilaginosa, and $R$. aeria were confirmed by a BLAST search.

\subsection{Development of a Novel One-Step Multiplex PCR Method Using Designed Primers}

Bacterial cells were cultured in BHI supplemented with 0.5\% yeast extract for 24 $\mathrm{h}$, and $1 \mathrm{ml}$ samples were then collected in microcentrifuge tubes and resuspended at a density of 1.0 McFarland standard [approximately $10^{7}$ colonyforming units $(\mathrm{CFU}) / \mathrm{ml}]$ in $1 \mathrm{ml}$ of sterile distilled water. A total of $5.6 \mu \mathrm{l}$ of the suspension was then used as the PCR template. The detection limit of PCR was assessed by serially diluting known numbers of bacterial cells in sterile distilled water and then subjecting each suspension to PCR. The multiplex PCR mixture contained $0.2 \mu \mathrm{M}$ of each primer, $10 \mu \mathrm{l}$ of $2 \times$ MightyAmp Buffer Ver.3 (Takara Bio Inc., Shiga, Japan), $0.4 \mu \mathrm{l}$ of MightyAmp DNA Polymerase (Takara), and 5.6 $\mu \mathrm{l}$ of the template in a final volume of $20 \mu \mathrm{l}$. PCRs were performed in a DNA thermal cycler (Applied Biosystems 2720 Thermal Cycler; Applied Biosystems, Forster City, CA, USA). PCR conditions included an initial denaturation step at $98^{\circ} \mathrm{C}$ for $2 \mathrm{~min}$, followed by 25 cycles consisting of $98^{\circ} \mathrm{C}$ for $10 \mathrm{~s}$ and $68^{\circ} \mathrm{C}$ for 1 min. PCR products were analyzed by $2.0 \%$ agarose gel electrophoresis and then 
visualized by electrophoresis in $1 \times$ Tris-borate-EDTA on a $2 \%$ agarose gel stained with ethidium bromide. A 100-bp DNA ladder (Takara Biomed, Shiga, Japan) was used as a molecular size marker. All experiments were performed in triplicate.

\subsection{Clinical Samples}

Sixty patients attending Nihon University Hospital, School of Dentistry at Matsudo, participated in the present study. They were divided into two subject groups: healthy implant (HI) and peri-implantitis (PI) groups. Thirty HI and thirty PI subjects were selected by inclusion criteria for peri-implantitis as follows: patients who underwent dental implantation treatments between 2015 and 2019; patients with at least one dental implant for more than half a year; according to the Guidelines of Periodontology, PI was defined as bleeding of probing (BOP) and/or probing pocket depth (PPD) $\geq 4 \mathrm{~mm}$, accompanied by bone tissue loss under the first thread of the implant (i.e., bone absorption $\geq 2 \mathrm{~mm}$ ). HI was defined as PPD $\leq 3 \mathrm{~mm}$, and the absence of BOP, pus discharge, and bone absorption. Exclusion criteria were as follows: patients with systematic diseases; patients receiving periodontal therapy within six months; taking immunosuppressive agents or antibiotics; the long-term use of contraceptive drugs; pregnant women.

Peri-implant sulcus fluid (PISF) samples were collected using endodontic paper points from all subjects and placed in a sterile microcentrifuge tube containing $1 \mathrm{ml}$ of Tris- $\mathrm{HCl}$ buffer (0.05 M, pH 7.2). Samples were dispersed by sonication for $30 \mathrm{~s}$ in an ice bath $(50 \mathrm{~W}, 20 \mathrm{kHz}$, Astrason System model XL 2020, NY., USA). Portions $(100 \mu \mathrm{l})$ of appropriate dilutions of these samples were plated, in triplicate, on Anaerobic Blood Agar (CDC), that consists of a Tryptic soy agar (Becton, Dickinson and Co., Sparks, MD, USA) supplemented with vi$\operatorname{tamin} \mathrm{K}_{1}$, hemin, L-cysteine, yeast extract, and sheep blood, and on selective medium plates that were developed for the isolation of oral Rothia species in our previous report [24]. Selective medium plates for oral Rothia species were cultured at $37^{\circ} \mathrm{C}$ for three days in an atmosphere of $5 \% \mathrm{CO}_{2}$ in a $\mathrm{CO}_{2}$ incubator. CDC plates for total cultivable bacteria were cultured at $37^{\circ} \mathrm{C}$ for five days under anaerobic conditions with a gas pack system. After the cultivation, the number of CFU was calculated. Also, the detection frequencies of red complex bacteria, i.e., P. gingivalis, Treponema denticola, and Tannerella forsythia in the remainder of each PISF sample were determined using PCR as previously described [28]. This study was approved by the Ethics Committee of Nihon University School of Dentistry at Matsudo, Japan (EC 19-033).

\subsection{Examination of Oxygen Concentrations that $\boldsymbol{R}$. aeria and Anaerobic Bacteria Needed for Growth in Vitro}

\subsubsection{Comparison of the Growth of R. aeria between Two Culture Conditions}

BHI agar plates on which $R$. aeria were inoculated were cultured at $37^{\circ} \mathrm{C}$ for three days under aerobic conditions and under anaerobic conditions with a gas 
pack system. After cultivation, the number of CFUs was calculated, and the CFUs of $R$. aeria under the two culture conditions were compared.

\subsubsection{Comparison of Growth in a Semi-Liquid Nutrient Medium between R. aeria and Anaerobic Bacteria}

A semi-liquid nutrient medium that consisted of $\mathrm{BHI}$ and $0.8 \%$ agar was prepared in a glass test tube. Bacterial suspensions of $R$. aeria JCM 11412 and $P$. gingivalis ATCC 33277 that were preincubated were inoculated into each medium with an inoculating needle and were cultured at $37^{\circ} \mathrm{C}$ for two days under aerobic conditions. After cultivation, the growth of $R$. aeria and $P$. gingivalis in semi-liquid nutrient medium was macroscopically compared.

\subsection{Statistical Analysis}

The numbers of oral Rothia species and total bacteria in the PISF samples from the HI and PI groups were compared using the Mann-Whitney $U$ test. Values of $\mathrm{P}<0.05$ were considered significant. The detection frequencies of red complex bacteria in both groups were compared using Fisher's exact test. Values of $\mathrm{P}<$ 0.05 were considered significant.

\section{Results}

\subsection{Multiplex PCR}

\subsubsection{Primer Design}

Six specific primers covering the upstream regions of the $16 \mathrm{~S}$ rDNA sequences of three oral Rothia species were designed in the present study (Figure 1). The specific forward primers were designated as RMFF for $R$. mucilaginosa, RDFF for $R$. dentocariosa, and RAFF for $R$. aeria, whereas the specific reverse primers were designated as RMFR for $R$. mucilaginosa, RDFR for $R$. dentocariosa, and RAFR for $R$. aeria. The amplicon sizes of $R$. mucilaginosa, $R$. dentocariosa, and $R$. aeria were $356 \mathrm{bp}, 541 \mathrm{bp}$, and $924 \mathrm{bp}$, respectively.

\subsubsection{Detection Limit}

Our one-step multiplex PCR method for identifying three oral Rothia species, $R$. mucilaginosa, $R$. dentocariosa, and $R$. aeria, successfully amplified DNA fragments of the expected sizes for each species (Figure 2). The detection limit was assessed in the presence of titrated bacterial cells, and the sensitivity of the PCR assay was between $5 \times 1$ and $5 \times 10 \mathrm{CFU}$ per PCR template $(5.0 \mu \mathrm{l})$ for the $R$. mucilaginosa-specific primer set with strain JCM 10910, the $R$. dentocariosaspecific primer set with strain JCM 306, and the $R$. aeria-specific primer set with strain JCM 11412 (data not shown).

\subsubsection{Assay of Representative Rothia Species and Representative Oral Bacteria}

The one-step multiplex PCR method used to identify $R$. mucilaginosa, $R$. dentocariosa, and $R$. aeria produced positive bands from each respective strain (Figure 1) and did not produce any amplicons from other Rothia species or with Streptococci, 


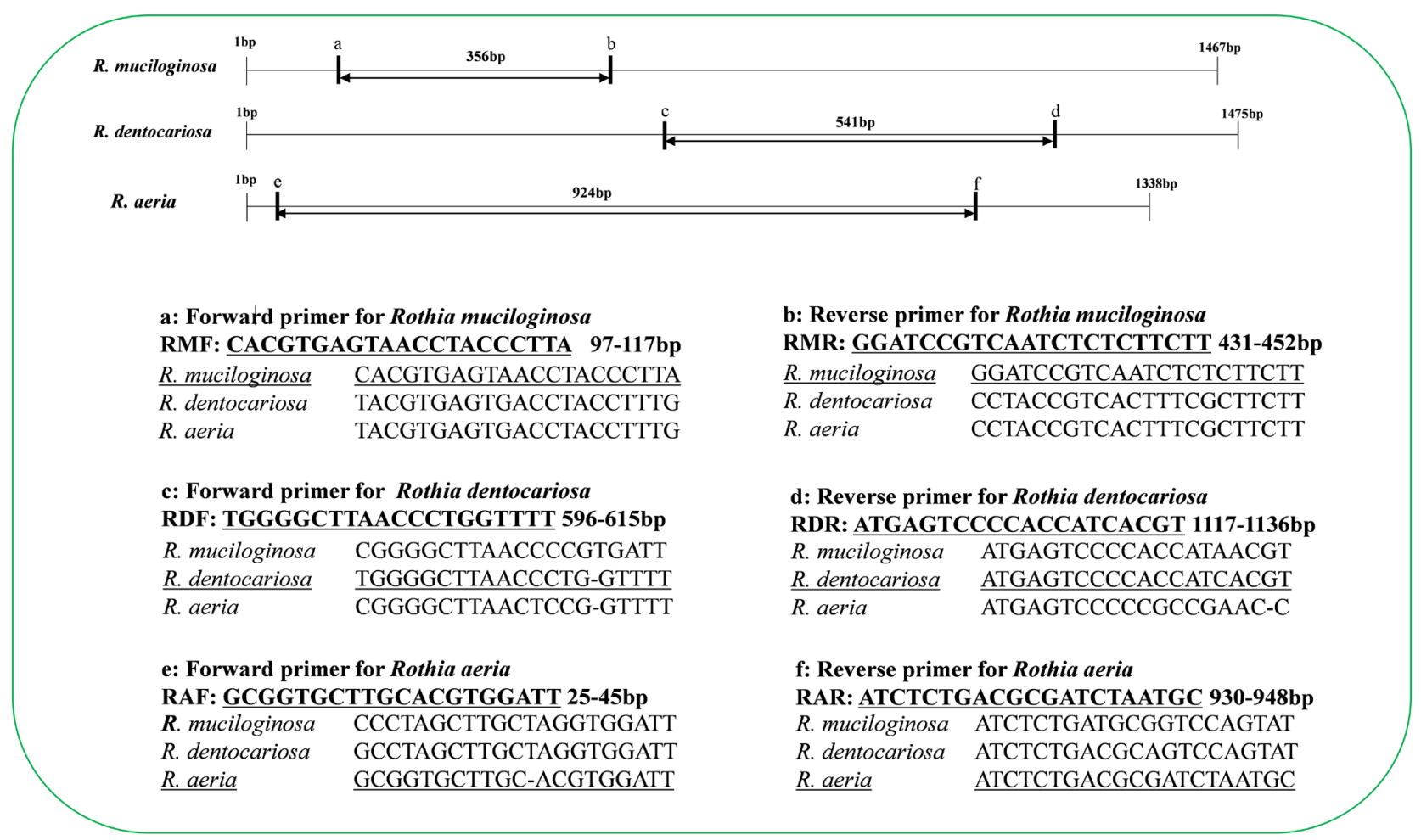

Figure 1. Locations and sequences of species-specific primers for 16S rDNA of oral Rothia species.

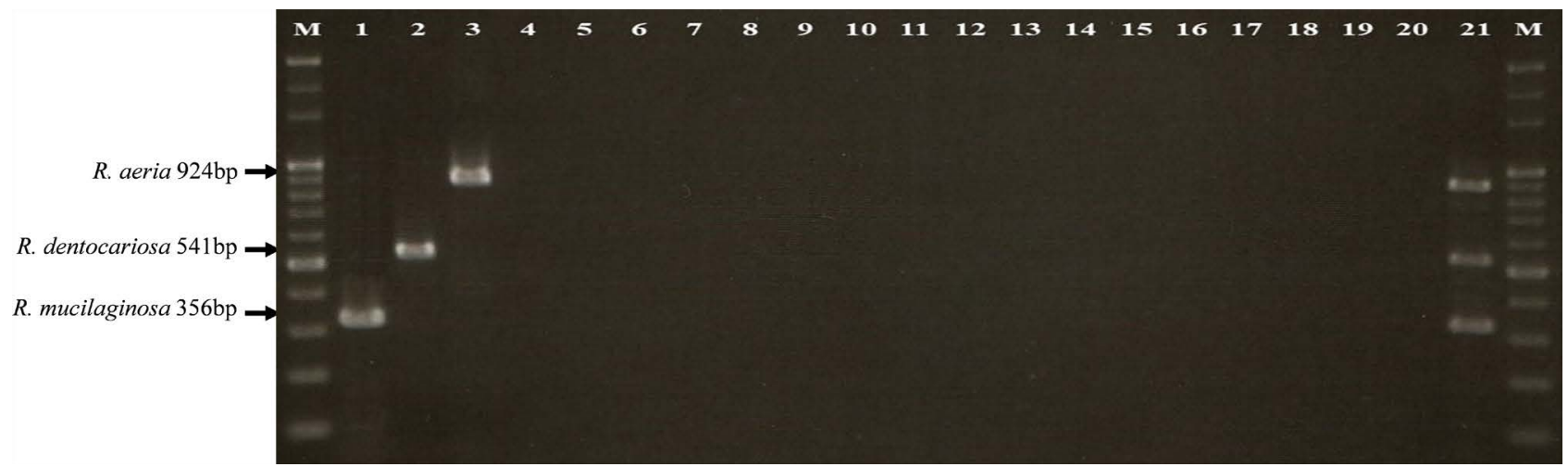

Lanes: $1: R$. mucilaginosa JCM 10910; 2: $R$. dentocariosa JCM 3067; 3: $R$. aeria JCM 11412; 4: $R$. terrae JCM 15158; 5: $R$. amarae JCM 11375; 6: $R$. nasimurium JCM 10909; 7: $R$. endophytica JCM 18541; 8: S. mitis ATCC 49456; 9: S. oralis ATCC 10557; 10: S. sanguinis ATCC 10556; 11: S. salivarius JCM 5707; 12: $S$. anginosus ATCC 33397; 13: $S$. mutans NCTC 10449; 14: A. naeslundii ATCC 12104; 15: A. oris ATCC 27044; 16: A. odontolyticus ATCC 17929; 17: A. israelii ATCC 12102; 18: C. matruchotii ATCC 14266; 19: C. durum ATCC 33449; 20: N. sicca ATCC 29256; 21: Mixture of $R$. mucilaginosa JCM 10910, $R$. dentocariosa JCM 3067, and $R$. aeria JCM 11412; M, molecular size marker (100-bp DNA ladder).

Figure 2. Multiplex PCR assay for identifying oral Rothia species.

Actinomyces, Neisseria, or Corynebacterium species used as representative oral bacteria with the designed primer sets. Moreover, three bands equivalent to $R$. mucilaginosa, $R$. dentocariosa, and $R$. aeria were produced from a mixed sample of the three oral Rothia species.

\subsection{Clinical Examination}

The clinical parameters of HI and PI groups are shown in (Table 1). The average 
ages and PPDs of HI and PI groups were 51 (range: 35 - 63) and 57 (range: 45 66), and $2.35 \mathrm{~mm}$ and $7.90 \mathrm{~mm}$, respectively. A comparison of the mean numbers of total bacteria and oral Rothia species between the two groups is shown in (Table 2). The mean number of total bacteria in the HI group was $1.24 \times 10^{6}$ CFU. The mean numbers of $R$. dentocariosa, $R$. mucilaginosa, and $R$. aeria in the HI group were $1.05 \times 10^{3} \mathrm{CFU}, 4.97 \times 10^{2} \mathrm{CFU}$, and $6.66 \times 10^{3} \mathrm{CFU}$, respectively. The mean number of total bacteria in the PI group was $7.16 \times 10^{6} \mathrm{CFU}$. The mean numbers of $R$. dentocariosa, $R$. mucilaginosa, and $R$. aeria in the PI group were $4.23 \times 10^{3} \mathrm{CFU}, 6.40 \times 10^{2} \mathrm{CFU}$, and $6.25 \times 10^{2} \mathrm{CFU}$, respectively. The mean number of $R$. aeria in PISF samples was significantly higher in the HI group than in the PI group $(\mathrm{P}<0.05)$.

A comparison of the proportions of oral Rothia species between the two groups is shown in (Figure 3). R. aeria in the PISF samples of the HI and PI groups was detected at $0.998 \%$ and $0.008 \%$, respectively, of total bacteria. $R$. mucilaginosa in the $\mathrm{HI}$ and PI groups was detected at $0.030 \%$ and $0.010 \%$, respectively, of total bacteria. $R$. dentocariosa in the $\mathrm{HI}$ and PI groups was detected at $0.176 \%$ and $0.079 \%$, respectively, of total bacteria. The proportion of $R$. aeria in PISF samples was significantly higher in the HI group than in the PI group $(\mathrm{P}<0.05)$.

The detection frequencies of red complex bacteria in PISF samples obtained from the two groups are shown in (Table 3). While the detection frequencies of all red complex bacteria, $P$. gingivalis, $T$. forsythia, and $T$. denticola, in PISF samples were significantly higher in the PI group than in the HI group $(\mathrm{P}<$ 0.01), these organisms were also detected from some of the HI group.

\subsection{Comparison of the Growth of R. aeria between Two Culture Conditions}

A comparison of the growth of $R$. aeria between two culture conditions is shown

Table 1. Clinical parameters of the two groups.

\begin{tabular}{ccccccc}
\hline & \multicolumn{2}{c}{ Subject } & \multicolumn{3}{c}{ Clinical findings } \\
\cline { 2 - 6 } Group & $\begin{array}{c}\text { No. of subjects } \\
\text { (male:female) }\end{array}$ & $\begin{array}{c}\text { Average age } \\
\text { (range) }\end{array}$ & BOP & $\begin{array}{c}\text { Pus } \\
\text { discharge }\end{array}$ & $\begin{array}{c}\text { Bone } \\
\text { loss }\end{array}$ & $\begin{array}{c}\text { Average PPD } \\
\text { (range) }\end{array}$ \\
\hline Healthy implants & 30 & 51 & - & - & - & $2.35 \mathrm{~mm}$ \\
Peri-implantitis & $(17: 13)$ & $(35-63)$ & & & $+3 \mathrm{~mm})$ \\
& 30 & 57 & + & + & + & $7.90 \mathrm{~mm}$ \\
$(14: 16)$ & $(45-66)$ & & & & & \\
\end{tabular}

Table 2. Comparison of the numbers of total bacteria and oral Rothia species between the two groups.

\begin{tabular}{lcccc}
\hline & $\begin{array}{c}\text { No. of } \\
\text { total bacteria }\end{array}$ & $\begin{array}{c}\text { No. of } \\
\text { R. dentocariosa }\end{array}$ & $\begin{array}{c}\text { No. of } \\
\text { R. mucilaginosa }\end{array}$ & $\begin{array}{c}\text { No. of } \\
\boldsymbol{R} \text {. aeria }\end{array}$ \\
\cline { 2 - 5 } & \multicolumn{4}{c}{$(\mathrm{CFU})$} \\
\hline Healthy implants $(\mathrm{n}=30)$ & $1.24 \times 10^{6}$ & $1.05 \times 10^{3}$ & $4.97 \times 10^{2}$ & $6.66 \times 10^{3 *}$ \\
Peri-implantitis $(\mathrm{n}=30)$ & $7.16 \times 10^{6}$ & $4.23 \times 10^{3}$ & $6.40 \times 10^{2}$ & $6.25 \times 10^{2 *}$ \\
\hline
\end{tabular}

${ }^{\star}$ Mann-Whitney $U$ test; $p<0.05$. 
in (Figure 4). $R$. aeria under aerobic conditions vigorously grew compared with under anaerobic conditions.

\subsection{Comparison of Growth in Semi-Liquid Nutrient Medium between $R$. aeria and Anaerobic Bacteria}

A comparison of growth in semi-liquid nutrient medium between $R$. aeria and anaerobic bacteria ( $P$. gingivalis) is shown in (Figure 5$). R$. aeria grew only at

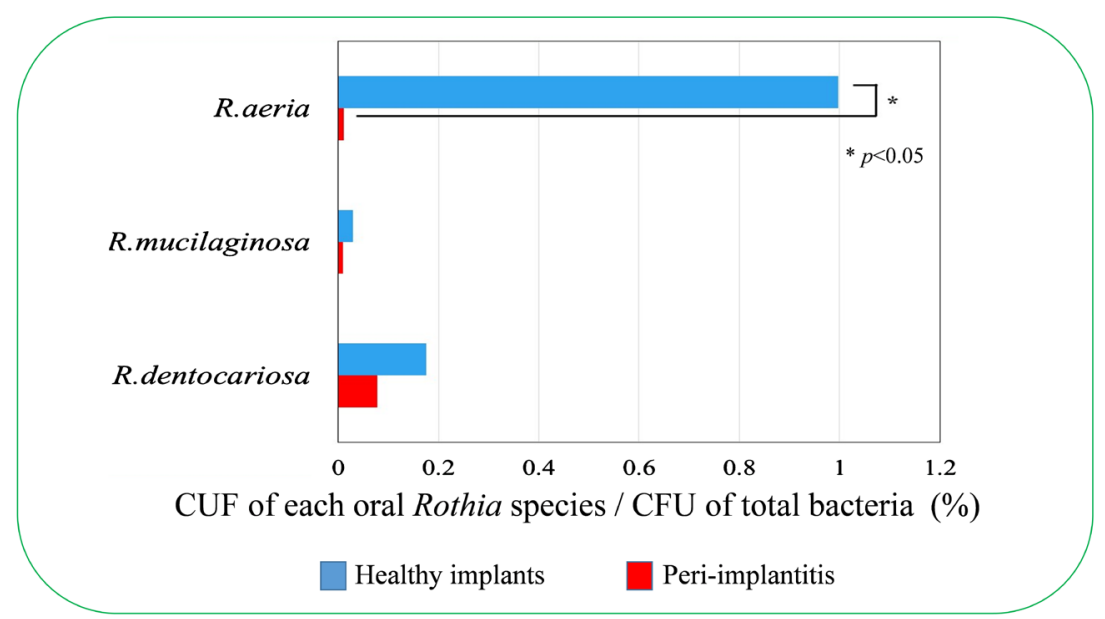

Figure 3. Comparison of the proportions of oral Rothia species between the two groups.

Table 3. Detection frequencies of red complex bacteria in PISF samples of the two groups.

\begin{tabular}{cccc}
\hline \multirow{2}{*}{ Species } & \begin{tabular}{r} 
Healthy implants $(\mathrm{n}=30)$ \\
\cline { 2 - 3 }
\end{tabular} & No. of positive samples $(\%$; frequency) & $\begin{array}{c}\text { Fisher's exact } \\
\text { test }\end{array}$ \\
\hline P. gingivalis & $6(20)$ & $24(80)$ & $p<0.01$ \\
T. forsythia & $8(27)$ & $23(77)$ & $p<0.01$ \\
T. denticola & $5(17)$ & $18(60)$ & $p<0.01$ \\
\hline
\end{tabular}

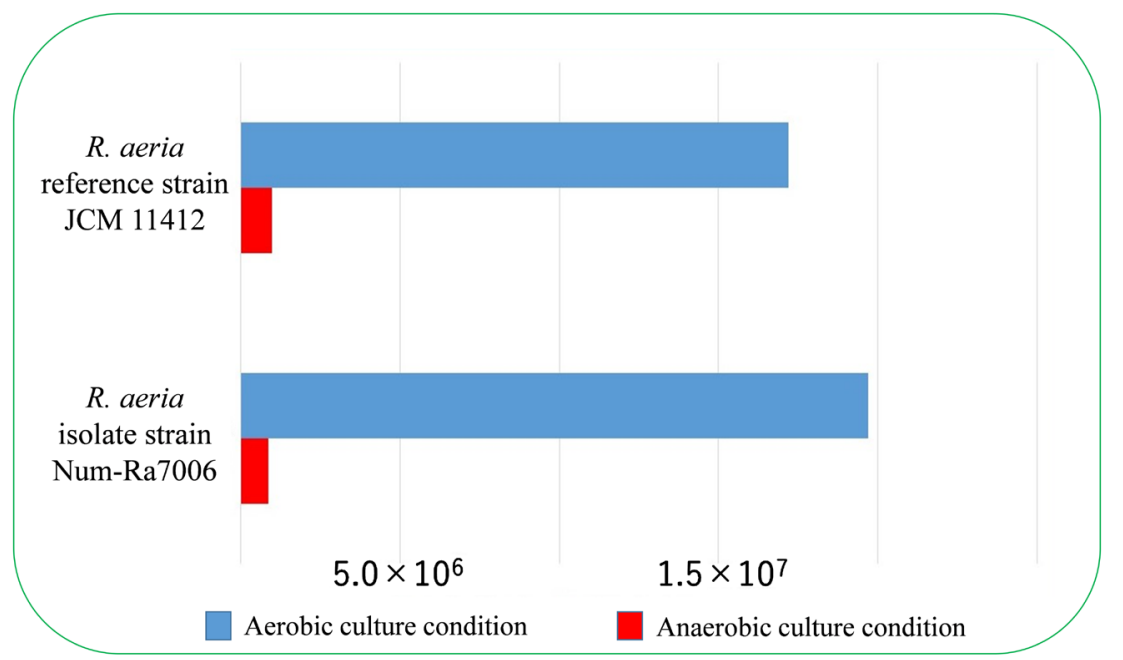

Figure 4. Comparison of growth of $R$. aeria between two culture conditions. 


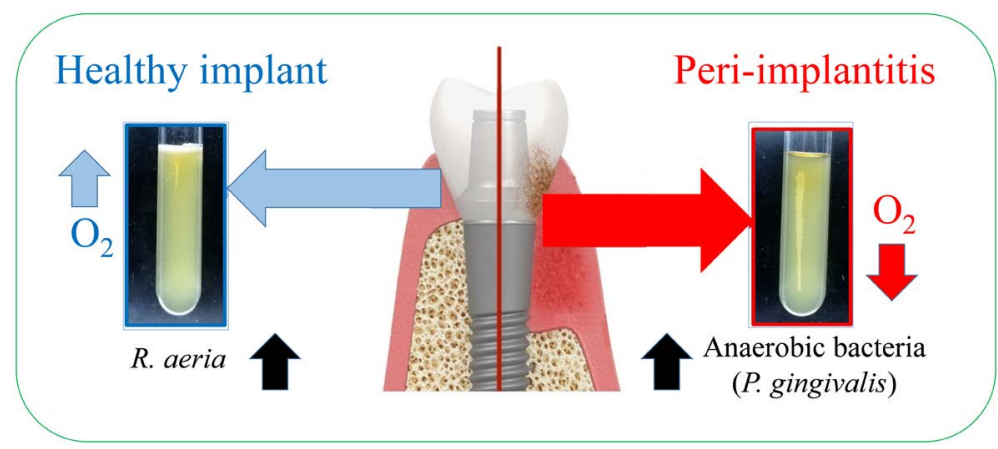

Figure 5. Comparison of growth in semi-liquid nutrient medium between $R$. aeria and anaerobic bacteria.

the upper layer where high oxygen concentrations existed. In contrast, $P$. gingivalis did not grow in the upper layer, but instead grew in the middle-under layer where little or no oxygen existed.

\section{Discussion}

The genus Rothia is Gram-positive and includes seven species, $R$. aeria, Rothia amarae, $R$. dentocariosa, Rothia endophytica, $R$. mucilaginosa, Rothia nasimurium, and Rothia terrae [24]. Among the Rothia, R. aeria, R. dentocariosa, and R. mucilaginosa inhabit the human oral cavity and pharynx. All three have been identified as opportunistic pathogens that can cause septicemia, endocarditis, and other serious infections [29] [30] [31]. $R$. aeria, which was originally classified as $R$. dentocariosa genomovar II, was first isolated from air and condensation water samples in the Russian space station Mir [32]. There was only one report in which $R$. aeria was detected in the mouths of healthy individuals [33] until our previous study demonstrated that $R$. aeria is part of the normal flora in the oral cavity [24].

Upon clinical microbiological examination, Rothia species can be mistaken for bacteria such as Dermabacter hominis, Actinomyces viscosus, Propionibacterium avidum, Corynebacterium matruchotii, and Nocardia spp., because many laboratories are unfamiliar with these organisms, which may be difficult to culture due to having the same gram-positive rods and to their varying aero-tolerance [34] [35] [36]. Moreover, some studies have previously reported that it is difficult to identify isolates, and that routine biochemical tests might misidentify $R$. aeria as $R$. dentocariosa [37] [38]. In addition, $R$. aeria can be mistaken for Nocardia spp. due to morphological similarities, and discrimination between $R$. aeria and Nocardia spp. needs further analyses, such as 16S rRNA sequencing [39]. Sequence analysis of several target genes is the most reliable method. However, it is expensive, laborious, and time-consuming. Thus, a simple and more reliable assay for identifying oral Rothia species is required.

In the present study, species-specific primers to identify oral Rothia species were designed using a one-step multiplex PCR method. These primers were able to distinguish $R$. mucilaginosa, $R$. dentocariosa, and $R$. aeria and did not react with representative oral bacteria or other Rothia species. Moreover, the novel 
one-step multiplex PCR analysis could directly use bacterial cells using MightyAmp DNA Polymerase Ver. 3 (Takara) and be completed in approximately 1.5 h. Our previous study also used a multiplex PCR method for the identification of oral Rothia species [24]. The previous method took approximately $2 \mathrm{~h}$; therefore, the new method was shorter by $30 \mathrm{~min}$.

In the present study, oral Rothia species were investigated as an indicator of healthy peri-implant tissue conditions. Currently, bacteriological examinations of implant treatments target periodontopathic bacteria such as red complex bacteria, which are detected qualitatively or quantitatively. However, those examinations do not precisely reflect the peri-implant tissue conditions, because periodontopathic bacteria may be detected at healthy peri-implant sites [23]. We have been searching for bacteria that are suitable as an indicator for healthy peri-implant tissue conditions. Recently, several studies have reported that oral Rothia species are detected more frequently in periodontally healthy subjects compared with chronic periodontitis patients [40] [41]. We therefore chose oral Rothia species, which are part of the normal oral flora, as possible indicator species in the present study. As a result, the mean number and proportions of $R$. aeria in PISF samples were significantly higher in the HI group than in the PI group ( $\mathrm{P}<0.05$ ); however, those of $R$. dentocariosa and $R$. mucilaginosa did not demonstrate significant differences between the groups. Moreover, the detection frequencies of all red complex bacteria were significantly higher in the PI group than in the HI group $(\mathrm{P}<0.01)$; however, these organisms were also detected in some samples of the HI group. Renvert et al. also reported that the prevalence of red complex bacteria, considered as key pathogens in periodontitis, is low and does not seem to differ by implant status [23]. In addition, $R$. aeria under aerobic conditions vigorously grew compared with anaerobic conditions and grew only at the upper layer where high oxygen concentrations existed in a semi-liquid nutrient medium. These results indicated that a shallow healthy peri-implant sulcus, where high oxygen concentration exists, is preferable for $R$. aeria growth.

We developed a one-step multiplex PCR method for the identification of oral Rothia species. The method described herein will be useful for determining the distribution and role of these organisms in various locations in humans. Moreover, the monitoring of $R$. aeria levels may be suitable as an indicator reflecting healthy peri-implant tissue conditions to aid in the prevention of peri-implantitis.

\section{Authors' Contributions}

Suzuki H, Tsuzukibashi O, Fukatsu A, corrected the data. Suzuki H wrote the manuscript. The concept of this manuscript was devised by Suzuki H. All authors read and approved the final manuscript.

\section{Conflicts of Interest}

The authors declare no conflicts of interest regarding the publication of this paper. 


\section{References}

[1] Albrektsson, T., Zarb, G., Worthington, P. and Eriksson, A.R. (1986) The LongTerm Efficacy of Currently Used Dental Implants: A Review and Proposed Criteria of Success. International Journal of Oral \& Maxillofacial Implants, 1, 11-25.

[2] Adell, R., Eriksson, B., Lekholm, U., Brånemark, P.I. and Jemt, T. (1990) LongTerm Follow-Up Study of Osseointegrated Implants in the Treatment of Totally Edentulous Jaws. International Journal of Oral \& Maxillofacial Implants, 5, 347-359.

[3] Arvidson, K., Bystedt, H., Frykholm, A., von Konow, L. and Lothigius, E. (1998) Five-Year Prospective Follow-Up Report of the Astra Tech Dental Implant System in the Treatment of Edentulous Mandibles. Clinical Oral Implants Research, 9, 225-234. https://doi.org/10.1034/j.1600-0501.1998.090403.x

[4] Henry, J., Laney, W.R., Jemt, T., Harris, D., Krogh, P.H.J., Polizzi, G., Zarb, G.A. and Herrmann, I. (1996) Osseointegrated Implants for Single-Tooth Replacement: A Prospective 5-Year Multicenter Study. International Journal of Oral \& Maxillofacial Implants, 11, 450-455.

[5] Lekholm, U., van Steenberghe, D., Herrmann, I., Bolender, C., Folmer, T., Gunne, J., Henry, P., Higuchi, K. and Laney, W.R. (1994) Osseointegrated Implants in the Treatment of Partially Edentulous Jaws: A Prospective 5-Year Multicenter Study. International Journal of Oral \& Maxillofacial Implants, 9, 627-635.

[6] Jemt, T., Chai, J., Harnett, J., Heath, R., Hutton, J.E., Johns, R.B., McKenna, S., McNamara, D.C., van Steenberghe, D., Taylor, R., Watson, R.M. and Herrmann, I. (1996) A 5-Year Prospective Multicenter Follow-Up Report on Overdentures Supported by Osseointegrated Implants. International Journal of Oral \& Maxillofacial Implants, 11, 291-298.

[7] Palmer, R.M., Smith, B.J., Palmer, P.J. and Froyd, P.D. (1997) A Prospective Study on Astra Single Tooth Implants. Clinical Oral Implants Research, 8, 173-179. https://doi.org/10.1034/j.1600-0501.1997.080303.x

[8] Mombelli, A. and Lang, N.P. (1998) The Diagnosis and Treatment of Peri-Implantitis. Periodontol 2000, 17, 63-76. https://doi.org/10.1111/j.1600-0757.1998.tb00124.x

[9] Hultin, M., Gustafsson, A., Hallstrom, H., Johansson, L.-A., Ekfeldt, A. and Klinge, B. (2002) Microbiological Findings and Host Response in Patients with PeriImplantitis. Clinical Oral Implants Research, 13, 349-358. https://doi.org/10.1034/j.1600-0501.2002.130402.x

[10] Quirynen, M., De Soete, M. and van Steenberghe, D. (2002) Infectious Risks for Oral Implants: A Review of the Literature. Clinical Oral Implants Research, 13, 1-19. https://doi.org/10.1034/j.1600-0501.2002.130101.x

[11] Roos-Jansaker, A.M., Renvert, S. and Egelberg, J. (2003) Treatment of Peri-Implant Infections: A Literature Review. Journal of Clinical Periodontology, 30, 467-485. https://doi.org/10.1034/j.1600-051X.2003.00296.x

[12] Roos-Jansaker, A.M., Lindahl, C., Renvert, H. and Renvert, S. (2006) Nine-to Fourteen-Year Follow up of Implant Treatment: I. Implant Loss and Associations to Various Factors. Journal of Clinical Periodontology, 22, 283-289. https://doi.org/10.1111/j.1600-051X.2006.00907.x

[13] Roos-Jansaker, A.M., Lindahl, C., Renvert, H. and Renvert, S. (2006) Nine-to Fourteen-Year Follow Up of Implant Treatment: II. Implant Loss and Associations to Various Factors. Journal of Clinical Periodontology, 33, 290-295. https://doi.org/10.1111/j.1600-051X.2006.00906.x

[14] Van der Weijden, G.A., van Bemmel, K.M. and Renvert, S. (2005) Implant Therapy in Partially Edentulous Periodontally Compromised Patients: A Review. Journal of 
Clinical Periodontology, 32, 506-511. https://doi.org/10.1111/j.1600-051X.2005.00708.x

[15] Haas, R., Haimbock, W., Mailath, G. and Watzek, G. (1996) The Relationship of Smoking and Peri-Implant Tissue: A Retrospective Study. Journal of Prosthetic Dentistry, 76, 592-596. https://doi.org/10.1016/S0022-3913(96)90435-7

[16] Lindquist, L.W., Carlsson, G.E. and Jemt, T. (1996) A Prospective 15-Year Follow-up Study of Mandibular Fixed Prostheses Supported by Osseointegrated Implants Clinical Results and Marginal Bone Loss. Clinical Oral Implants Research, 7, 329-336. https://doi.org/10.1034/j.1600-0501.1996.070405.x

[17] Esposito, M., Hirsch, J.M., Lekholm, U. and Thomsen, P. (1998) Biological Factors Contributing to Failures of Osseointegrated Oral Implants (II). Etiopathogenesis. European Journal of Oral Sciences, 106, 721-764. https://doi.org/10.1046/j.0909-8836..t01-6-.x

[18] Baelum, V. and Ellegard, B. (2004) Implant Survival in Periodontally Compromised Patients. Journal of Periodontology, 75, 1404-1412. https://doi.org/10.1902/jop.2004.75.10.1404

[19] van Winkelhoff, A.J., Goene, R.J., Benschop, C. and Folmer, T. (2000) Early Colonization of Dental Implants by Putative Periodontal Pathogens in Partially Edentulous Patients. Clinical Oral Implants Research, 11, 511-520.

https://doi.org/10.1034/j.1600-0501.2000.011006511.x

[20] Quirynen, M., Vogels, R., Peeters, W., van Steenberghe, D., Naert, I. and Haffajee, A. (2006) Dynamics of Initial Subgingival Colonization of Pristine Peri-Implant Pockets. Clinical Oral Implants Research, 17, 25-37. https://doi.org/10.1111/j.1600-0501.2005.01194.x

[21] Leonhardt, A., Renvert, S. and Dahlen, G. (1999) Microbial Findings at Failing Implants. Clinical Oral Implants Research, 10, 339-345. https://doi.org/10.1034/j.1600-0501.1999.100501.x

[22] Aas, J.A., Paster, B.J., Stokes, L.N., Olsen, I. and Dewhirst, F.E. (2005) Defining the Normal Bacterial Flora of the Oral Cavity. Journal of Clinical Microbiology, 43, 5721-5732. https://doi.org/10.1128/JCM.43.11.5721-5732.2005

[23] Renvert, S., Roos-Jansaker, A.M., Lindahl, C., Renvert, H. and Persson, G.R. (2007) Infection at Titanium Implants with or without a Clinical Diagnosis of Inflammation. Clinical Oral Implants Research, 18, 509-516. https://doi.org/10.1111/j.1600-0501.2007.01378.x

[24] Tsuzukibashi, O., Uchibori, S., Kobayashi, T., Umezawa, K., Mashimo, C., Nambu, T., Saito, M., Hashizume-Takizawa, T. and Ochiai, T. (2017) Isolation and Identification Methods of Rothia Species in Oral Cavities. Journal of Microbiological Methods, 134, 21-26. https://doi.org/10.1016/j.mimet.2017.01.005

[25] Lang, N.P., Brägger, U., Walther, D., Beamer, B. and Kornman, K.S. (1993) Ligature-Induced Peri-Implant Infection in Cynomolgus Monkeys. I. Clinical and Radiographic Findings. Clinical Oral Implants Research, 4, 2-11.

https://doi.org/10.1034/j.1600-0501.1993.040101.x

[26] Charalampakis, G., Rabe, P., Leonhardt, A. and Dahlén, G. (2011) A Follow-Up Study of Peri-Implantitis Cases after Treatment. Journal of Clinical Periodontology, 38, 864-871. https://doi.org/10.1111/j.1600-051X.2011.01759.x

[27] de Waal, Y.C., Raghoebar, G.M., Meijer, H.J., Winkel, E.G. and van Winkelhoff, A.J. (2016) Prognostic Indicators for Surgical Peri-Implantitis Treatment. Clinical Oral Implants Research, 27, 1485-1491. https://doi.org/10.1111/clr.12584

[28] Ashimoto, A., Chen, C., Bakker, I. and Slots, J. (1996) Polymerase Chain Reaction Detection of 8 Putative Periodontal Pathogens in Subgingival Plaque of Gingivitis 
and Advanced Periodontitis Lesions. Oral Microbiology and Immunology, 11, 266-273. https://doi.org/10.1111/j.1399-302X.1996.tb00180.x

[29] Tarumoto, N., Sujino, K., Yamaguchi, T., Umeyama, T., Ohno, H., Miyazaki, Y. and Maesaki, S. (2012) A First Report of Rothia aeria Endocarditis Complicated by Cerebral Hemorrhage. Internal Medicine, 51, 3295-3299.

https://doi.org/10.2169/internalmedicine.51.7946

[30] Bousquet, A., Soler, C., Martinaud, C., Join-Lambert, O. and Malfuson, J.V. (2011) Pneumoniae and Rothia dentocariosa. Médecine et Maladies Infectieuses, 41, 621-622. https://doi.org/10.1016/j.medmal.2011.07.011

[31] Kaasch, A.J., Saxler, G. and Seifert, H. (2011) Septic Arthritis Due to Rothia mucilaginosa. Infection, 39, 81-82. https://doi.org/10.1007/s15010-010-0065-5

[32] Li, Y., Kawamura, Y., Fujiwara, N., Naka, T., Liu, H., Huang, X., Kobayashi, K. and Ezaki, T. (2004) Rothia aeria sp. nov., Rhodococcus baikonurensis sp. nov. and Arthrobacter russicus sp. nov., Isolated from Air in the Russian Space Laboratory Mir. International Journal of Systematic and Evolutionary Microbiology, 54, 827835. https://doi.org/10.1099/ijs.0.02828-0

[33] Haraszthy, V.I., Zambon, J.J., Sreenivasan, P.K., Zambon, M.M., Gerber, D., Rego, R. and Parker, C. (2007) Identification of Oral Bacterial Species Associated with Halitosis. Journal of the American Dental Association, 138, 1113-1120. https://doi.org/10.14219/jada.archive.2007.0325

[34] Brown, J.M., Georg, L.K. and Waters, L.C. (1969) Laboratory Identification of Rothia dentocariosa and its Occurrence in Human Clinical Materials. Applied and Environmental Microbiology, 17, 150-156.

https://doi.org/10.1128/am.17.1.150-156.1969

[35] Binder, D., Zbinden, R., Widmer, U., Opravil, M. and Krause, M. (1997) Native and Prosthetic Valve Endocarditis Caused by Rothia dentocariosa: Diagnostic and Therapeutic Considerations. Infection, 25, 22-26. https://doi.org/10.1007/BF02113502

[36] Funke, G., von Graevenitz, A., Clarridge 3rd, J.E. and Bernard, K.A. (1997) Clinical Microbiology of Coryneform Bacteria. Clinical Microbiology Reviews, 10, 125-159. https://doi.org/10.1128/CMR.10.1.125

[37] Verrall, A.J., Robinson, P.C., Tan, C.E., Mackie, W.G. and Blackmore, T.K. (2010) Rothia aeria as a Cause of Sepsis in a Native Joint. Journal of Clinical Microbiology, 48, 2648-2650. https://doi.org/10.1128/JCM.02217-09

[38] Michon, J., Jeulin, D., Lang, J.M. and Cattoir, V. (2010) Rothia aeria Acute Bronchitis: the First Reported Case. Infection, 38, 335-337.

https://doi.org/10.1007/s15010-010-0012-5

[39] Uni, M., Shinohara, A., Nukina, A., Nakamura, F., Nannya, Y., Mizoguchi, M., Okazaki, M., Tatsuno, K. and Moriya, K. (2015) Successful Management of Rothia aeria Pneumonia during the Peritransplant Period: First Reported Case in Allogeneic Hematopoietic Stem Cell Transplant. Leukemia \& Lymphoma, 56, 248-250. https://doi.org/10.3109/10428194.2014.916799

[40] Abusleme, L., Dupuy, A.K., Dutzan, N., Silva, N., Burleson, J.A., Strausbaugh, L.D., Gamonal, J. and Diaz, P.I. (2013) The Subgingival Microbiome in Health and Periodontitis and its Relationship with Community Biomass and Inflammation. The ISME Journal, 7, 1016-1025. https://doi.org/10.1038/ismej.2012.174

[41] Kistler, J.O., Booth, V., Bradshaw, D.J. and Wade, W.G. (2013) Bacterial Community Development in Experimental Gingivitis. PLoS ONE, 8, e71227. https://doi.org/10.1371/journal.pone.0071227 